\title{
Comparación de la Dosis Absorbida Determinada por Dosimetría Directa con los Parámetros del Tomógrafo Computarizado del HPGDR
}

\author{
Erika Magaly Tierra Vilema, \\ Blanca Fabiola Pumalema Morocho,
}

Facultad de Ciencias, Escuela Superior Politécnica de Chimborazo,

Riobamba, Ecuador

Myrian Cecilia Borja Saavedra,

Facultad de Ciencias, CIDED, Escuela Superior Politécnica de Chimborazo, Riobamba, Ecuador

William Bladimir Cevallos Cevallos,

Facultad de Ciencias, Escuela Superior Politécnica de Chimborazo,

Riobamba, Ecuador

Marina Leonor Bonilla Lucero,

Docente de la Facultad de Ciencias Pecuarias, Escuela Superior Politécnica de Chimborazo, Riobamba, Ecuador

Doi: 10.19044/esj.2019.v15n18p152 URL:http://dx.doi.org/10.19044/esj.2019.v15n18p152

\section{Resumen}

El objetivo de la investigación fue comparar la relación existente entre la dosis de radiación obtenida del dosímetro, con las cantidades dosimétricas del equipo en tomografías de cráneo simple en el Hospital Provincial Regional General Docente Riobamba, durante el período de febrero a abril del 2018. Para la exploración se utilizó el tomógrafo Toshiba TSX-031A de 16 cortes helicoidal y un dosímetro electrónico de lectura directa DoseRAE2, a 3 centímetros de la nuca del paciente y se controló la dosis suministrada mediante el monitor de consola del operador. La investigación se realizó con 48 pacientes adultos con un intervalo de edad de 18 a 60 años. Los datos fueron analizados mediante R-Studio y Microsoft Excel. Se aplicó el test de Kolmogorov Smirnov y la prueba de $\mathrm{t}$ de Student. Los resultados indicaron que los valores medios del tomógrafo tienen una emisión de radiación excesiva de $0,59 \mathrm{mSv}$ sobre el valor de referencia según la Comunidad Europea y el Colegio Americano de Radiología; conociendo así la dosis a la cual se irradia al paciente. Se recomienda la calibración de los parámetros dosimétricos del equipo para evitar una sobredosis y los efectos biológicos en el paciente. 
Palabras clave: Biofísica, radiodiagnóstico, tomografía computarizada de cráneo, magnitudes dosimétricas, dosimetría en tc.

\title{
Comparison of the Absorbed Dose Determined by Direct Dosimetry with the Parameters of the HPGDR Computed Tomographer
}

\section{Erika Magaly Tierra Vilema, Blanca Fabiola Pumalema Morocho,}

Facultad de Ciencias, Escuela Superior Politécnica de Chimborazo,

Riobamba, Ecuador

Myrian Cecilia Borja Saavedra,

Facultad de Ciencias, CIDED, Escuela Superior Politécnica de Chimborazo,

Riobamba, Ecuador

William Bladimir Cevallos Cevallos,

Facultad de Ciencias, Escuela Superior Politécnica de Chimborazo,

Riobamba, Ecuador

\section{Marina Leonor Bonilla Lucero,}

Docente de la Facultad de Ciencias Pecuarias, Escuela Superior Politécnica de Chimborazo, Riobamba, Ecuador

\begin{abstract}
This paper focuses on comparing the relationship between the dose of radiation obtained from the dosimeter with the dosimetric quantities of the equipment in single skull tomography in the Provincial General Regional Hospital Riobamba, during the period from February to April 2018. The scan was carried out using the Toshiba TSX-031A 16-helical slice tomograph and a DoseRAE2 direct-reading electronic dosimeter. This was placed 3 centimeters from the patient's nape, and the delivered dose was controlled by the operator's console monitor. The research was conducted with 48 adult patients with an age range of 18 to 60 years. The data was analyzed using RStudio and Microsoft Excel. The Kolmogorov Smirnov test and the Student $t$ test were applied. The results indicated that the average values of the tomograph have an emission of excessive radiation of $0.59 \mathrm{mSv}$ over the reference value according to the European Community and the American College of Radiology; knowing thus the dose to which the patient is
\end{abstract}


irradiated. It is recommended to calibrate the dosimetric parameters of the equipment to avoid an overdose and the biological effects on the patient.

Keywords: Biophysics, radiodiagnosis, skull computed tomography, dosimetric magnituudes, ct dosimetry

\section{Introduction}

El CTDI representa el promedio de la dosis absorbida a lo largo del eje $\mathrm{z}$ de una serie de exposiciones contiguas. Esta ésta se calcula dividiendo la integral de la dosis absorbida por el grosor o espesor del corte (T) de la sección estudiada (Giraldo \& Clavijo, 2008, pp. 22-23). Su unidad es el mGy y permite conocer con precisión la dosis de cada corte.

Para ajustar el equipo se utiliza el CTDI100 que corresponde a un segmento de $100 \mathrm{~mm}$ y el cual emplea una cámara de ionización en forma de lápiz y fantomas de acrílico estandarizados (Giraldo \& Clavijo, 2008, pp. 22 23). Las mediciones del CTDI en un fantoma estándar de cabeza o cuerpo pueden ser utilizadas para proporcionar una indicación del CTDI promedio sobre el corte tomográfico producido en una exploración axial (Valdez, 2006, pp. 32-35).

$$
\mathrm{CTDI}_{100}=\frac{1}{\mathrm{NT}} \int_{5 \mathrm{~cm}}^{5 \mathrm{~cm}} \mathrm{D}_{(\mathrm{z})} \mathrm{dz}
$$

La radiología es un área de la medicina que permite obtener imágenes del cuerpo con la finalidad de identificar anomalías y lesiones que expliquen los síntomas como parte del proceso del diagnóstico. Permite además efectuar intervenciones con fines de tratamiento al guiar los procedimientos e incluso usar las radiaciones como tratamiento (Andrade, 2014).Se la conoce también como radiodiagnóstico o diagnóstico por imagen (Arevalo, 2011).

La dosis en TC está determinada según las características de fabricación del tomógrafo, así como el protocolo, la elección de la técnica ( $\mathrm{mA}$ y kV), la longitud del barrido de estudio, la talla, peso, edad y región anatómica a explorar. La dosis en el paciente y la dosis efectiva depende de la calidad del haz de rayos $\mathrm{X}$, por lo cual la dosis está relacionada linealmente con el producto de la corriente (mA) y el tiempo de examinación, mientras que la dosis absorbida por una parte del cuerpo depende inversamente del pitch para un $\mathrm{mA}$ constante $\mathrm{y}$ así poder satisfacer el requerimiento diagnóstico. Los parámetros técnicos y operacionales que son aplicados tanto en la tomografía convencional como helicoidal se detallan en la Tabla 4-2 (Valdez, 2006, pp. 35-36).

En 1970 el físico Hounsfield desarrolló un corte tomográfico sectorial sobre la cabeza de un ser humano. En octubre de 1971 en los Estados Unidos (EEUU) se instala el primer equipo de tomografía computarizada (TC) especializado en imágenes craneales (Hrescak, 2012), el cual ha ido 
evolucionando considerablemente con el desarrollo de los detectores y reduciendo el tiempo de exposición, dando inicio a la revolución en el radiodiagnóstico. Las magnitudes dosimétricas en TC son útiles para conocer el daño de la radiación y determinar las medidas de protección radiológica para los pacientes.

El término tomografía tienen su origen en las palabras griegas tomos (corte, rebanada o sección) y grafos (imagen), por lo cual es un procedimiento con imágenes que usa un equipo especial de rayos $\mathrm{X}$ para crear imágenes detalladas de las regiones internas del cuerpo como los órganos, huesos y otros tejidos en una "rebanada" delgada. Se le llama también tomografía axial computarizada (TAC) (NIH, 2013).

El principio fundamental de la tomografía es el movimiento del tubo de rayos $\mathrm{X}$ en una dirección, el cual gira $360^{\circ}$ mientras la placa radiográfica se mueve en dirección opuesta. Por tal motivo, una superficie plana de la anatomía humana es perfectamente visible mientras que las áreas por encima o por debajo quedan borradas (Vásquez, 2011, pp. 15-16).

El Hospital Provincial Regional General Docente Riobamba (HPGDR) es una institución pública con alta demanda de pacientes. Cuenta con el área de imagenología la cual cuenta con diversos equipos de radiodiagnóstico como es el tomógrafo computarizado Toshiba TSX-031A de tipo helicoidal de 16 cortes que se puso en funcionamiento a partir de abril del 2009; sin embargo, no se hace uso adecuado del control de dosis de radiación absorbida para los diferentes estudios que se realizan los pacientes, como sucede en el caso de los estudios de Tomografía Computarizada helicoidal de Cráneo simples (TCC simple). En este sentido, esta investigación tiene como objetivo comparar la dosis absorbida determinada por dosimetría directa en pacientes a través de los parámetros del equipo en estudios de cráneo para el Tomógrafo Computarizado (TC) TOSHIBA TSX -031A del HPGDR que permitirá conocer si los valores son los mismos o hay un rango de diferencia entre ellos. De igual forma, se tiene como objetivo saber si los valores de la dosimetría del equipo se encuentran calibrados y si están o no dentro de los valores de referencia según el comité de la Comunidad Europea (CE), el Colegio Americano de Radiología (ACR) y la Sociedad Radiológica de América del Norte (RSNA).

\section{Material y Método}

La investigación fue de tipo experimental, observacional y descriptiva. Los datos fueron obtenidos mediante la radiación directa al paciente y generada por el dosímetro electrónico DoseRAE 2 y un tomógrafo. Los resultados fueron almacenados y analizados a fin de conocer si la dosimetría del equipo ha sido correctamente calibrada y si los pacientes recibieron la dosis absorbida adecuada dentro de los límites recomendados 
por los organismos internacionales de control, como lo establece la guía de calidad en TC de la CE.

La población de estudio representa a los pacientes que se realizaron tomografías computarizadas helicoidales de cráneo simple (TCC simple) en el HPGDR; la muestra estadística tiene un nivel de confianza del 95\%, con un error de precisión de tipo II equivalente al 5\%. La muestra es intencionada y está compuesta de 48 pacientes que no presentaron situación crítica, con edades entre 18 y 60 años de edad, de género masculino y femenino.

$$
\begin{gathered}
n=\frac{Z^{2} \times p \times q}{e^{2}} \\
n=\frac{(1,96)^{2} \times 0,5 \times 0,5}{(0,141)^{2}}=48.3
\end{gathered}
$$

Tabla 1. Características de la muestra

\begin{tabular}{|c|c|c|c|c|}
\hline Género & Total & $\begin{array}{c}\text { De } 18 \text { a } \\
31 \text { años }\end{array}$ & $\begin{array}{c}\text { De 32 a } \\
45 \text { años }\end{array}$ & $\begin{array}{c}\text { De 46 a } \\
60 \text { años }\end{array}$ \\
\hline Mujeres & 29 & 13 & 10 & 6 \\
\hline Hombres & 19 & 7 & 6 & 6 \\
\hline $\begin{array}{c}\text { Total de } \\
\text { pacientes }\end{array}$ & 48 & 20 & 16 & 12 \\
\hline
\end{tabular}

Fuente: Pacientes del HPGDR.

Elaborado por: Tierra Erika, 2018.

Los resultados de los pacientes fueron obtenidos en el servicio de radiología del HPGDR en la sala de tomografía, donde el procedimiento más influyente fue la aplicación al paciente de la dosis para el estudio de TCC simple. Los datos fueron obtenidos en días laborables, en un lapso aproximado de 3 meses, en horario de $09 \mathrm{~h} 00$ a 17h00. Para asegurar la representatividad de los resultados de los pacientes, se tuvieron en cuenta parámetros como la edad e historial clínico que permitió estimar las dosis para los 48 pacientes adultos. Se utilizó el tomógrafo computarizado (TC) Toshiba TSX-031A de 16 cortes helicoidal y el dosímetro electrónico calibrado de lectura directa DoseRAE 2, con las siguientes características:

\begin{tabular}{|l|l|}
\hline Marca y modelo & $\begin{array}{l}\text { Tomógrafo Computarizado } \\
\text { Toshiba Activion de } 16 \text { cortes }\end{array}$ \\
\hline Modo & Helicoidal \\
\hline Velocidad & $0,75 \mathrm{seg}$ a $10 \mathrm{seg}$ por rotación \\
\hline Tensión & $80 \mathrm{kV}$ a $140 \mathrm{kV}$ \\
\hline Corriente & $10 \mathrm{~mA}$ a $500 \mathrm{~mA}$ \\
\hline
\end{tabular}




\begin{tabular}{|l|l|}
\hline $\begin{array}{l}\text { Marca y modelo del } \\
\text { tubo de rayos X }\end{array}$ & TSX $-031^{\mathrm{a}}$ \\
\hline Espesor de corte & $0,5 \mathrm{~mm}$ a $10,0 \mathrm{~mm}$ \\
\hline Fecha de instalación & Abril de 2009 \\
\hline
\end{tabular}

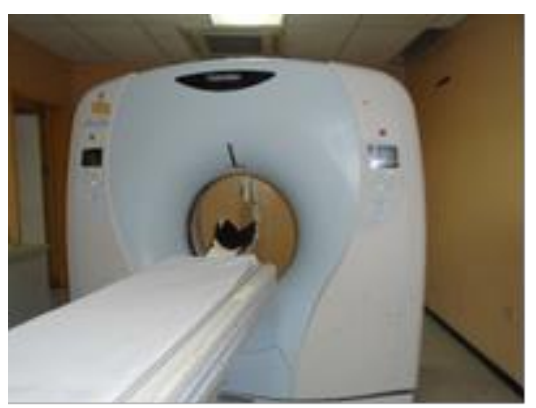

Figura 1. Características del Tomógrafo Computarizado Toshiba Activion TSX-031A del HPGDR

\begin{tabular}{|l|l|}
\hline Marca / modelo & Dosímetro electrónico personal DoseRAE 2 / PRM-1200 \\
\hline Detección & Rayos X y rayos gamma \\
\hline Sensores & $\begin{array}{l}\text { Doble canal que emplea un diodo semiconductor y un cristal de } \\
\text { centelleo }\end{array}$ \\
\hline Lee directamente & Dosis equivalente y tasa de dosis equivalente \\
\hline Tamaño & $85 \mathrm{~mm}$ x $55 \mathrm{~mm}$ x $9,6 \mathrm{~mm}$ \\
\hline Peso & 1,8 onzas $(50 \mathrm{mg})$ \\
\hline Rango dosis & $0 \mu \mathrm{Sv}$ a $10 \mathrm{~Sv}$ \\
\hline Rango tasa de dosis & $0.01 \mu \mathrm{Sv} / \mathrm{h}$ a $10 \mathrm{~Sv} / \mathrm{h}$ \\
\hline Exactitud de la dosis & $\pm 15 \%$ \\
\hline Respuesta energética & $20 \mathrm{keV}$ a $6 \mathrm{MeV}$ \\
\hline $\begin{array}{l}\text { Temperatura } \\
\text { ambiente }\end{array}$ & $-4{ }^{\circ} \mathrm{F}$ a $122{ }^{\circ} \mathrm{F} \mathrm{o}-20{ }^{\circ} \mathrm{C}$ a $50^{\circ} \mathrm{C}$ \\
\hline Tipo de Batería & $\mathrm{LIR} 2450$ recargable \\
\hline Fecha de calibración & $\mathrm{Septiembre} 09,2016$ \\
\hline
\end{tabular}

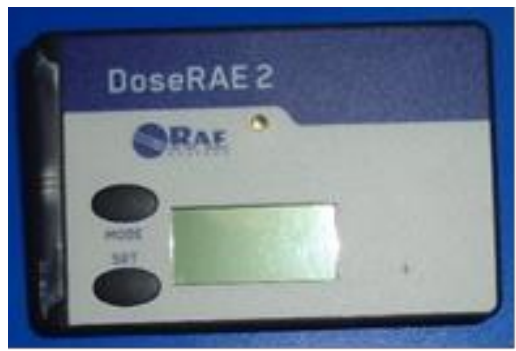

Figura 2. Características del dosímetro electrónico de lectura directa DoseRAE 2 del HPGDR 
La exploración craneal por Tomografía Computarizada se realizó mediante un examen médico de diagnóstico no invasivo ni doloroso que reproduce múltiples imágenes del interior del cuerpo por la combinación de un equipo de rayos $X$.Dicha exploración se puede observar a través de un monitor o a su vez imprimirse (Vásquez, 2011).

Las imágenes captadas mediante la emisión de radiación son generadas desde un tubo de rayos X, el mismo gira alrededor de la cabeza del paciente. La radiación es absorbida por el paciente y el resto son almacenados por una fila de detectores.

Por otra parte, la mesa se desplaza con un giro de exploración de $360^{\circ}$, lo que permite obtener múltiples proyecciones de imágenes claras y con detalles de visualización de los órganos internos, huesos, tejidos blandos o vasos sanguíneos; a partir de lo cual se pueden analizar y diagnosticar lesiones de la cabeza como derrames y tumores cerebrales que no pueden ser observados en una radiografía convencional (rayos X) (Vásquez., 2011).

En la exploración se consideró el angular del gantry de $10^{\circ}$ a $12^{\circ}$ por encima de la línea orbito-meatal para reducir la exposición de los lentes oculares (Hofer, 2008). El paciente fue colocado sobre la mesa en posición boca arriba con el cuello en extensión, reposando su cabeza en el cabezal del equipo. Se utilizó un sistema de luces como guía para el correcto posicionamiento.

Los datos del paciente tales como: número de cédula, historia clínica, apellidos, nombres completos, sexo y edad fueron registrados en el equipo. Luego, para proceder con la exploración se seleccionó el protocolo de estudio denominado técnica de cráneo helicoidal.

El dosímetro DoseRAE 2 fue colocado a $3 \mathrm{~cm}$ debajo de la nuca de cada paciente, para obtener la lectura de la dosis equivalente. El tomógrafo, al tener su propia dosimetría y ser esta calculada automáticamente, permitió conocer la dosis suministrada al paciente mediante los indicadores del índice de Dosis en Tomografía Computarizada Volumétrico (CTDIvol) y el Producto de la Dosis por la Longitud (DLP).

Tabla 2. Datos recolectados de un estudio de TCC simple de modo helicoidal

\begin{tabular}{|c|c|c|c|c|c|c|}
\hline \multirow[b]{2}{*}{ Paciente } & \multirow[b]{2}{*}{ Edad } & \multirow{2}{*}{$\begin{array}{c}\text { Dosímetro } \\
\text { Dosis } \\
\begin{array}{c}\text { Equivalente } \\
(\mathrm{mSv})\end{array}\end{array}$} & \multicolumn{4}{|c|}{$\begin{array}{l}\text { Tomógrafo Computarizado Toshiba TSX - } \\
\text { 031A }\end{array}$} \\
\hline & & & $\begin{array}{l}\text { CTDIvol } \\
(\mathbf{m G y})\end{array}$ & $\begin{array}{c}\text { DLP } \\
(\mathbf{G y} \cdot \mathrm{Cm})\end{array}$ & $\begin{array}{l}\text { Longitud } \\
\quad(\mathrm{mm})\end{array}$ & $\begin{array}{c}\mathbf{N}^{\circ} \text { de } \\
\text { imágenes }\end{array}$ \\
\hline $\mathrm{P} 1$ & 58 & 8,868 & 81,0 & 1,84 & 170,0 & 35 \\
\hline P 2 & 50 & 9,073 & 81,0 & 1,52 & 160,0 & 33 \\
\hline P 3 & 29 & 6,075 & 81,0 & 1,35 & 140,0 & 29 \\
\hline P 4 & 33 & 8,688 & 81,0 & 1,27 & 130,0 & 27 \\
\hline P 5 & 21 & 7,680 & 81,0 & 1,39 & 145,0 & 30 \\
\hline P 6 & 37 & 2,540 & 78,7 & 1,55 & 170,0 & 35 \\
\hline P 7 & 21 & 9,304 & 81,0 & 1,35 & 140,0 & 29 \\
\hline
\end{tabular}




\begin{tabular}{|c|c|c|c|c|c|c|}
\hline P 8 & 47 & 9,380 & 81,0 & 1,60 & 170,0 & 35 \\
\hline P 9 & 46 & 2,319 & 82,3 & 1,50 & 155,0 & 32 \\
\hline P 10 & 60 & 15,740 & 78,7 & 1,39 & 150,0 & 31 \\
\hline P 11 & 38 & 4,120 & 81,0 & 1,43 & 150,0 & 31 \\
\hline P 12 & 36 & 6,225 & 81,0 & 1,27 & 130,0 & 27 \\
\hline P 13 & 39 & 7,321 & 81,0 & 1,27 & 130,0 & 27 \\
\hline P 14 & 35 & 6,390 & 81,0 & 1,39 & 145,0 & 30 \\
\hline P 15 & 18 & 5,710 & 81,0 & 1,39 & 145,0 & 30 \\
\hline P 16 & 25 & 6,030 & 81,0 & 1,35 & 140,0 & 29 \\
\hline P 17 & 18 & 7,180 & 81,0 & 1,39 & 145,0 & 30 \\
\hline P 18 & 18 & 7,157 & 81,0 & 1,19 & 120,0 & 25 \\
\hline P 19 & 44 & 6,127 & 81,0 & 1,39 & 145,0 & 30 \\
\hline P 20 & 51 & 7,058 & 81,0 & 1,52 & 160,0 & 33 \\
\hline P 21 & 26 & 12,970 & 81,0 & 1,43 & 150,0 & 31 \\
\hline P 22 & 28 & 6,944 & 81,0 & 1,15 & 115,0 & 24 \\
\hline P 23 & 43 & 7,605 & 81,0 & 1,35 & 140,0 & 29 \\
\hline P 24 & 34 & 4,363 & 81,0 & 1,43 & 150,0 & 31 \\
\hline P 25 & 21 & 7,506 & 81,0 & 1,35 & 140,0 & 29 \\
\hline P 26 & 35 & 6,756 & 81,0 & 1,35 & 140,0 & 29 \\
\hline P 27 & 43 & 4,690 & 81,0 & 1,35 & 140,0 & 29 \\
\hline P 28 & 27 & 6,806 & 78,7 & 1,39 & 150,0 & 31 \\
\hline P 29 & 32 & 6,607 & 81,0 & 1,35 & 140,0 & 29 \\
\hline P 30 & 28 & 6,494 & 81,0 & 1,35 & 140,0 & 29 \\
\hline P 31 & 29 & 5,083 & 81,0 & 1,23 & 125,0 & 26 \\
\hline P 32 & 18 & 5,933 & 81,0 & 1,31 & 135,0 & 28 \\
\hline P 33 & 21 & 7,018 & 81,0 & 1,35 & 140,0 & 29 \\
\hline P 34 & 21 & 16,530 & 81,0 & 1,56 & 165,0 & 34 \\
\hline P 35 & 23 & 6,843 & 81,0 & 1,31 & 135,0 & 28 \\
\hline P 36 & 43 & 8,806 & 81,0 & 1,48 & 155,0 & 32 \\
\hline P 37 & 32 & 6,305 & 81,0 & 1,35 & 140,0 & 29 \\
\hline P 38 & 29 & 6,053 & 81,0 & 1,35 & 140,0 & 29 \\
\hline P 39 & 58 & 7,302 & 78,7 & 1,31 & 140,0 & 29 \\
\hline P 40 & 47 & 7,290 & 78,7 & 1,31 & 140,0 & 29 \\
\hline P 41 & 50 & 8,033 & 81,0 & 1,27 & 130,0 & 27 \\
\hline P 42 & 46 & 8,031 & 81,0 & 1,48 & 155,0 & 32 \\
\hline P 43 & 30 & 13,520 & 81,0 & 1,43 & 150,0 & 31 \\
\hline P 44 & 22 & 4,172 & 81,0 & 1,39 & 145,0 & 30 \\
\hline P 45 & 53 & 4,829 & 81,0 & 1,43 & 150,0 & 31 \\
\hline P 46 & 39 & 6,370 & 81,0 & 1,31 & 150,0 & 31 \\
\hline P 47 & 36 & 5,290 & 81,0 & 1,43 & 135,0 & 28 \\
\hline P 48 & 53 & 9,574 & 82,3 & 1,37 & 140,0 & 29 \\
\hline F & Rep & 170909670 & \\
\hline
\end{tabular}

Fuente: Reporte del tomógrafo del estudio de TCC simple de modo helicoidal.

Elaborado por: Tierra Erika, 2018. 


\section{Análisis de Datos}

Los datos obtenidos fueron filtrados mediante un diagrama de cajas y bigotes, describiendo la dispersión y simetría. Además, los datos fueron graficados y analizados mediante el programa R-Studio, obteniéndose un total de 43 datos del DLP del tomógrafo y 42 datos de la dosis absorbida del dosímetro.

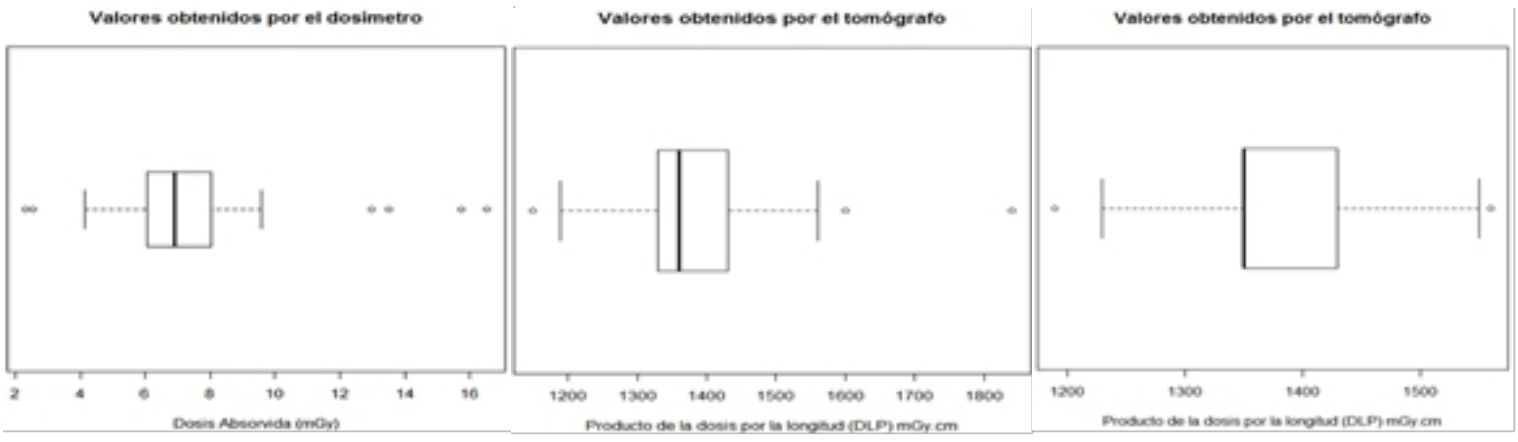

Figura 3. Diagrama de cajas y bigotes de los datos

Tabla 3. Datos atípicos de las dosis absorbidas en estudios de TCC simple

\begin{tabular}{|c|c|}
\hline $\begin{array}{c}\text { Datos atípicos de la Dosis } \\
\text { absorbida mSv }\end{array}$ & $\begin{array}{c}\text { Datos atípicos del } \\
\text { DLP mGy.cm }\end{array}$ \\
\hline 2,54 & 1840 \\
\hline 2,319 & 1600 \\
\hline 15,74 & 1150 \\
\hline 12,97 & 1190 \\
\hline 16,53 & 1560 \\
\hline 13,52 & \\
\hline
\end{tabular}

Fuente: Reporte de tomógrafo de dosis absorbidas en estudios de TCC simple.

Elaborado por: Tierra Erika, 2018.

Al aplicar la prueba de normalidad mediante el test de Kolmogorov Smirnov, con un nivel de significancia $(\alpha)$ de 0.05 y con un nivel de confianza de 95\%, tenemos que el valor crítico de la dosis absorbida fue de 0,20517; mientras que el valor crítico del DLP fue de 0,20283 , lo que permitió realizar los cálculos y gráficas mediante el programa R-Studio.

Tabla 4. Valores de la prueba de normalidad de los datos de la dosis absorbida

\begin{tabular}{|c|c|c|}
\hline \multicolumn{3}{|c|}{ Prueba de Kolmogorov Smirnov (D) } \\
\hline Variables & Valor de D & p-value \\
\hline Dosis absorbida & 0,080 & 0,9292 \\
\hline DLP & 0,152 & 0,2725 \\
\hline
\end{tabular}



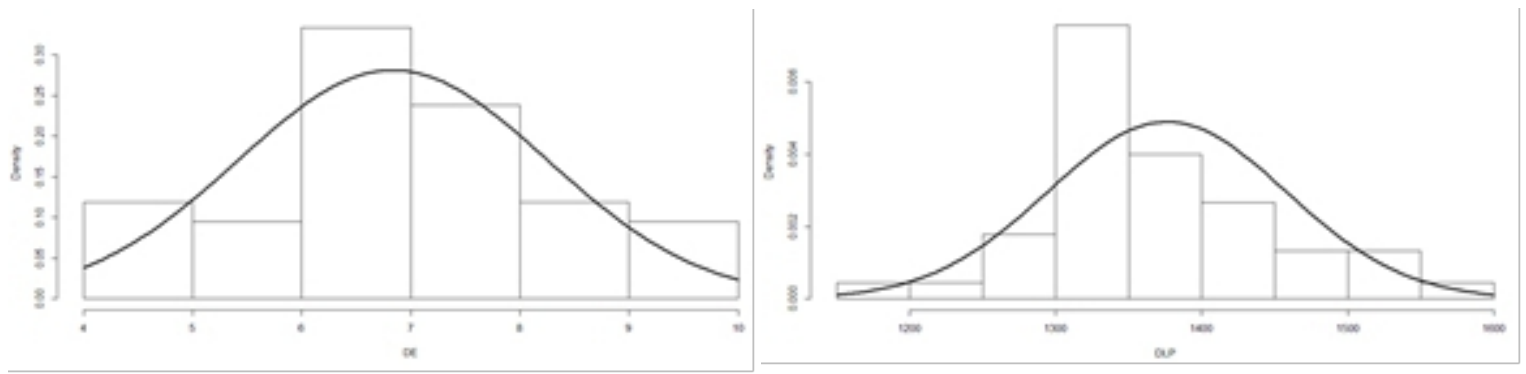

Figura 5. Curva de normalidad de la dosis absorbida obtenida por el dosímetro y el tomógrafo.

Para el cálculo de los valores de las dosis efectivas tanto para el dosímetro (Ec 2) como para el tomógrafo (Ec 3) se utilizaron las siguientes ecuaciones:

$$
\begin{aligned}
& E=\sum_{T} W_{T} \times H_{T} \\
& E=D L P \times E_{D L P}
\end{aligned}
$$

Al conocer los valores medios a través de Microsotf Excel 2016, se analizaron los datos obtenidos y se aplicó la prueba t de Student con la prueba de la varianza:

Tabla 5. Resultados de la Prueba F de las varianzas

\begin{tabular}{|l|c|c|}
\hline \multicolumn{2}{|c|}{ Prueba F para varianzas de dos muestras } \\
\hline Parámetros & $\begin{array}{c}\text { Dosímetro } \\
\text { Dosis efectiva mSv }\end{array}$ & Tomógrafo Dosis efectiva mSv \\
\hline Media & 4,92 & 2,89 \\
\hline Varianza & 1,04 & 0,03 \\
\hline Observaciones i & 42,00 & 45,00 \\
\hline Grados de libertad & 41,00 & 44,00 \\
\hline F & \multicolumn{2}{|c|}{35,57} \\
\hline $\mathrm{P}(\mathrm{F}<=$ f) una cola & & $3,79 \mathrm{E}-23$ \\
\hline Valor crítico para F (una cola) & \\
\hline
\end{tabular}

Fuente: Resultados de la Prueba $F$ de las varianzas mediante Excel. Elaborado por: Tierra Erika, 2018.

Tabla 6. Resultados de la prueba t de las dos muestras

\begin{tabular}{|l|c|c|}
\hline \multicolumn{3}{|c|}{ Prueba t para dos muestras suponiendo varianzas desiguales } \\
\hline Parámetros & $\begin{array}{c}\text { Dosímetro Dosis } \\
\text { efectiva mSv }\end{array}$ & $\begin{array}{c}\text { Tomógrafo Dosis } \\
\text { efectiva mSv }\end{array}$ \\
\hline Media & 4,92 & 2,89 \\
\hline Varianza & 1,04 & 0,03 \\
\hline Observaciones & 42,00 & 45,00 \\
\hline Diferencia hipotética de las medias & \multicolumn{3}{|c|}{0,00} \\
\hline Grados de libertad & \multicolumn{3}{|c|}{43,00} \\
\hline
\end{tabular}




\begin{tabular}{|l|c|}
\hline Estadístico $\mathrm{t}$ & 12,73 \\
\hline $\mathrm{P}(\mathrm{T}<=\mathrm{t})$ una cola & $1,77 \mathrm{E}^{-16}$ \\
\hline Valor crítico de $\mathrm{t}$ (una cola) & 1,68 \\
\hline $\mathrm{P}(\mathrm{T}<=\mathrm{t})$ dos colas & $3,53 \mathrm{E}^{-16}$ \\
\hline Valor crítico de $\mathrm{t}$ (dos colas) & 2,02 \\
\hline
\end{tabular}

Fuente: Microsoft Excel - Resultados de la prueba t de las dos muestras.

Elaborado por: Tierra Erika, 2018.

Dado que el estadístico $\mathrm{T}=12,73$ es mayor que el valor crítico que es de 1,68 y que la probabilidad de estadístico en el caso bilateral (dos colas) toma el valor de 3,53E-16 es menor a $0,025(0,05 / 2=0,025)$, se pudo asumir que el estadístico está dado al azar; por tanto, se afirma que las medias presentan diferencias estadísticamente significativas.

\section{Resultado}

El CTDI es la principal cantidad dosimétrica en TC, ya que es considerado un buen índice de exposición, siendo utilizado para determinar el DLP y a partir de esta la dosis efectiva; tomando como referencia los parámetros dosimétricos del tomógrafo que se visualizan en el monitor de la consola.

El valor máximo y mínimo del CTDI vol que es de 82,3 - 78,7 mGy con un rango de 3,6 mGy es mayor al valor recomendado por el Colegio Americano de Radiología el cual es de 60 mGy (Andisco, Blanco, \& Buzzi, 2014).

Para el caso de TCC simple, el valor máximo y mínimo del DLP es de 1840 - 1150 mGy.cm con un rango de 690 mGy, observándose de la misma manera que es mayor al valor de referencia según la Comunidad Europea que es de 1050 mGy.cm (Andisco, Blanco, \& Buzzi, 2014).

En lo que respecta a la comparación de medias de las dosis efectivas obtenidas mediante las mediciones del dosímetro electrónico DoseRAE 2 y de los parámetros observados en la consola del tomógrafo en estudios de TCC simple, el análisis estadístico muestra que existen diferencias de las dosis efectivas y son 4,92 $\mathrm{mSv}$ (dosímetro) y 2,89 $\mathrm{mSv}$ (tomógrafo).

Según la literatura el comité ACR y la RSNA dan a conocer que el valor de referencia de la dosis efectiva, tanto para las magnitudes dosimétricas en órgano como para la dosimetría en TC, es de $5 \mathrm{mSv}$ en órgano y $2,3 \mathrm{mSv}$ en TC craneal (RadiologyInfo.org, 2017).

\section{Conclusión}

Las cantidades dosimétricas en TC (CTDIvol, DLP) están relacionadas a la dosis absorbida, las mismas están determinadas por las 
características propias del tomógrafo que dependen de la edad del paciente, así como de la región anatómica explorada del protocolo y la técnica usada.

Al evaluar los valores de las dosis absorbidas por el paciente de la radiación emitida por el tomógrafo computarizado (TC) Toshiba TSX-031A y el dosímetro electrónico DoseRAE 2 con respecto a los límites establecidos por la Comunidad Europea (CE) y el Colegio Americano de Radiología (ACR), se obtuvo que para el tomógrafo son CTDIvol de 60 mGy y el DLP 1050 mGy.cm para cráneo de rutina (TC de cráneo simple), mientras que el ACR y la Sociedad Radiológica de América del Norte (RSNA) dan a conocer el valor límite de la dosis efectiva para el tomógrafo es de 2,3 mSv en TC de cráneo y para el dosímetro electrónico es de $5 \mathrm{mSv}$ en médula ósea.

Al realizar la comparación entre los valores medios de las dosis efectivas tanto del tomógrafo como del dosímetro electrónico, se mostró una diferencia estadística, la cual permite evaluar los riesgos de la radiación en los pacientes como la posibilidad de desarrollar cáncer en un determinado tiempo, teniendo en cuenta la sensibilidad de los tejidos u órganos a la radiación.

El desconocimiento de la información completa del paciente como es peso, talla, edad, y la falta de mantenimiento técnico del equipo (calibración del tomógrafo) al realizar el examen, pueden ser atenuantes que inciden para que este reciba mayor cantidad de radiación.

\section{References:}

1. Andalucía, F. d. (2009). Principios de la tomografia computarizada. Revista digital para profesionales de la enseñanza. (4), 1-2.

2. Andisco, D., B., S., \& Buzzi, A. (2014). Dosimetría en radiología. Elsevier España, 78(2), 114-117.

3. Arevalo, E. Radiologia. Blogger. [En línea] 16 de Febrero de 2011. [Consulta: 15 de Agosto de 2017.] http://estefaarevalo18.blogspot.com/2011/02/definicion.html.

4. Giraldo R, J. C., \& Clavijo A. (2008). "Tomografía computarizada por rayos $X$ : fundamentos y actualidad". Ingeniería Biomédica. Vol. 2. $\mathrm{n}^{\circ}$ 4, págs. 13-31.

5. Hofer, M. (2008). Fundamentos físicos y técnicos de TC; Dosis radiactiva. En A. Alcocer (Ed.), Manual Practico de TC (Quinta ed., págs. 6-12; 174-175). Buenos Aires: Panamericana. Recuperado el 20 de Marzo de 2018

6. Hrescak, D. M. (2012). HISTORIA Godfrey Newbold Hounsfield: historia e impacto de la tomografía computada. SciELO, 76(4), 331341.

7. RadiologyInfo.org. (2017). Dosis de radiación en exámenes de rayos $X \quad C T$. Image Gently. Obtenido de https://translate.googleusercontent.com/translate_c?depth=1\&hl=es \& 
prev=search\&rurl=translate.google.com.ec\&sl=en\&sp=nmt $4 \& u=h t t p$ s://www.radiologyinfo.org/en/info.cfm\%3Fpg\%3Dsafetyxray\&xid=17259,1500003,15700022,15700105,15700124,15700149, 15700168,15700173

8. Toshiba Medical Systems Corporation (2007). Informacion sobre el escáner Toshiba Activion TSX-031A. En T. M. Corporation, Manual de utilización del escáner Toshiba Activion TSX-031A (págs. 42-180). Recuperado el 21 de Marzo de 2018

9. Valdez T., \& Müsel J., (2006). Tomografía multidetector-16 de cráneo en niños: Estimación de dosis a órganos críticos por DTL. México: Universidad Autónoma Metropolitana-Iztapalapa, págs. 1-58.

10. Vásquez., J. G. (2011). La Tomografía y Exploración de la cabeza por TAC para el paciente. En J. G. Vásquez., Manual Práctico de Tomografia. (Cuarta ed., págs. 15-18; 239-240). Recuperado el 21 de Marzo de 2018, de http://www.academia.edu/10780497/MANUAL_PRACTICO_DE_T OMOGRAFIA 